\title{
基于影像学的ARDS诊疗进展: 从形态到功能
}

\author{
程卫 ${ }^{1 \dagger}$, 何怀武 ${ }^{\dagger \dagger}$, 苏龙翔 ${ }^{1}$, 崔娜 ${ }^{1}$, 王小亭 ${ }^{1}$, 周翔 ${ }^{1}$, 翁利 ${ }^{2}$, 隆云 $^{1^{*}}$, 杜斌, 刘大为 ${ }^{1}$
}

1. 中国医学科学院\&北京协和医学院, 北京协和医院, 疑难重症及罕见病国家重点实验室, 重症医学科, 北京 100730;

2. 中国医学科学院\&北京协和医学院, 北京协和医院, 疑难重症及罕见病国家重点实验室, 内科ICU, 北京 100730

$\dagger$ 同等贡献

*联系人, E-mail: 1y_icu@aliyun.com

收稿日期: 2021-06-12; 接受日期: 2021-07-17; 网络版发表日期: 2021-08-16

北京市科技计划课题(首都临床诊疗技术研究及转化应用ARDS循环保护通气策略的建立和示范应用)(批准号: Z201100005520051), 首都卫生 发展科研专项(首发2020-2-40111)和2020年北京临床重点专科卓越项目(批准号: ZK128001)资助

摘要 急性呼吸客迫综合征(acute respiratory distress syndrome, ARDS)概念被提出的50年来, 其争议和挑战不断. 肺部影像学的进步见证了ARDS的发展变迁, 从最早的胸部X线片到胸部断层扫描(computed tomography, CT), 再 到无放射性、床旁的胸部超声、电阻抗成像等方法, 在ARDS的诊断和治疗以及对病理生理机制的研究中起到重 要的作用. ARDS影像学发展从整体深入到局部, 从肺通气深入到肺血流灌注, 从单纯的呼吸评估到与循环评估相 结合, 从形态分析发展到功能评估. ARDS影像学不仅被应用于诊断, 更侧重床旁连续评估以指导治疗. 本文综述 了目前临床可用的ARDS影像学评估进展, 以期更好地理解ARDS的形态和功能改变, 更好地指导临床治疗以改 善患者预后.

关键词急性呼吸蹇迫综合征, 胸部X线片, 胸部断层扫描, 肺部超声, 胸部电阻抗断层成像

急性呼吸若迫综合征(acute respiratory distress syndrome, ARDS)迄今已有 50 年的历史, 在近半个世 纪的发展长河中, 伴随着不断的争议和挑战, 但在争 议和挑战中, ARDS的诊治不断突破, 不断进步. 现今 的ARDS, 已不是单纯的肺部病变诊断, 而是一组具有 某些病理生理性的临床特征的综合征 ${ }^{[1 \sim 3]}$. 肺部影像学 也从形态深入到功能, 从整体深入到局部, 从肺通气深 入到肺血流, 从单纯的呼吸评估到与循环评估相结合, 从形态诊断到功能监测指导治疗 ${ }^{[4]}$. 现今, 肺部影像学 更侧重对机械通气效果的监测, 对肺复张、俯卧位等 潜能的评估以及对肺血流的监测等形态结合功能更加
系统的全面评估. 本文拟对ARDS相关诊断和指导治 疗的影像学方法进行阐述.

\section{1 胸部X线片}

ARDS的概念直到1967年才真正被提出, 它定义 了ARDS典型的呼吸困难、顽固性低氧的临床表现, 双肺弥漫性阴影的X线特征和肺泡塌陷、间质与肺泡 水肿及透明膜形成的病理特征 ${ }^{[5]}$. 此后, ARDS的定义 几经演变, 直到2012年柏林定义 ${ }^{[6]}$ 的提出, 大家才对 ARDS的认识达成一致, 影像学方面也明确了胸部X线

引用格式: 程卫, 何怀武, 苏龙翔, 等. 基于影像学的ARDS诊疗进展: 从形态到功能. 中国科学: 生命科学, 2021, 51: 963-969

Cheng W, He H W, Su L X, et al. Advances on acute respiratory distress syndrome based on imaging: from morphology to function (in Chinese). Sci Sin Vitae, 2021, 51: 963-969, doi: 10.1360/SSV-2021-0201 
片(chest X-ray CXR)的标准, 即双肺浸润性病变, 不能 完全用心源性肺水肿、胸腔积液、肺不张和肺结节等 解释 ${ }^{[7]}$.

CXR用于ARDS的诊断面临诸多问题. 即使在高 收入国家, ARDS仍然被低估, 近期一项纳入 50 个国家 459 个ICU的观察性研究发现, 重度ARDS的识别率为 $78.5 \%$, 而轻度ARDS仅有 $51.3 \%{ }^{[8]}$, 对ARDS诊断不足 的很大一部分原因是对支持其诊断的 CXR浸润影的 性质认识不统一. 片状、浸润影不伴肺门影增宽是 ARDS的典型表现，但临床上经常发现CXR表现为不 对称、重力依赖区偶尔以肺叶为主的病变. 人工智能 在一定程度上解决了识别不足的问题，但由于算法不 成熟，尚未得到广泛应用和验证 ${ }^{[7,9]}$. 另外, CXR在 ARDS起病2 3天后的典型渗出期才会有所表现，并且 对于胸腔积液、肺实变和肺泡间质综合征的诊断准确 率要明显低于断层扫描(computed tomography, CT)和 肺部超声, 这也是ARDS早期诊断率低的原因 ${ }^{[10,11]}$. CXR也被用于肺部的功能评估，Wallet等人 ${ }^{[12]}$ 利用压 力变化过程中肺的密度变化来监测肺复张效果, 得出 了与压力-容积(pressure-volume, PV)曲线良好的相关 性, 但这种方法需要专用软件将CXR进行数字化处理, 过程复杂, 无法常规应用.

CXR表现作为ARDS概念的一部分, 促进了人们 对该综合征的认识和重视，但随着技术的进步，其不 足也表现得越来越明显. CXR仅为粗略的形态学评估, 仅提供有或无等定性问题，不能定量评估气体分布变 化, 也无法进行深入的肺功能评估, 在ARDS诊治中的 地位日趋降低.

\section{2 胸部断层扫描}

ARDS的柏林定义允许使用 CT 来诊断肺部阴影. 早在1980年代CT就用于描述ARDS病变的不均一性, 其典型表现为从背侧到腹侧的不均匀病变, 包括正常 通气肺组织、通气不良的肺组织和无通气的肺组 织 ${ }^{[13]}$. CT 的应用为ARDS的病理生理提供了新的见解, 并且证明了ARDS患者肺实质病变的不均一性 ${ }^{[4,14]}$. 实 际上与CXR类似, CT下ARDS可以表现为不同的形态, 弥漫性、局灶性甚至肺叶为主的病变均可出现 ${ }^{[15]}$. 但 与CXR相比, CT能更准确地反映病变肺区域的大小, 定量CT扫描还能测量肺部病变的体积或重量. 研究发
现，肺部钝性创伤中肺挫伤体积大于总肺体积的 $20 \%$, 具有良好的敏感性和特异性来预测ARDS的发生 ${ }^{[16,17]}$. 胸部CT可用于ARDS肺部功能的评估. 首先, CT 可用以评估不同类型机械通气时肺通气的变化. Yoshida等人 ${ }^{[18]}$ 发现，与压力支持通气相比，气道压力 释放通气可以显著减少非通气区域, 增加正常通气区 域. 其次, CT可以预测ARDS患者肺复张的潜能. CT下 的肺部形态可以显著影响呼气末正压(end-expiratory positive pressure, PEEP)、肺复张(recruitment maneuver，RM)、俯卧位等的效果. 弥漫性通气缺失患者与 局灶病变相比，在增加PEEP或RM后可以获得更大程 度的复张和更长时间的氧合改善 ${ }^{[19]}$. CT下可以用复张 后不通气区域的减少来评估肺复张的效果, Gattinoni 等人 ${ }^{[4,20]}$ 发现，复张前后非通气肺实质重量下降 $9 \%$ 可 以很好预测肺复张潜能. 然而, 既往几项利用CT下不 同方法计算肺复张潜能的研究发现, RM或应用PEEP 后, 可复张肺组织的数量变化很大, 并且用于肺复张 的定量 CT扫描需要专用的软件在每个 CT图像中手动 描绘肺的周长, 临床操作复杂, 耗时耗力. 最后, CT可 以根据肺应变测量局部的应力大小. 应变是指肺组织 在跨肺压作用下产生的形变, 肺组织产生的反作用力 称为应力. 在均质组织中, 施加的力使各部分产生相 同的应变, 而ARDS病变的不均一性产生了相邻组织 的应变差, Cressoni等人 ${ }^{[21]}$ 发现, ARDS肺组织局部的 应力可能接近施加压力的两倍, 并且随着ARDS的严 重程度, 这种病变的范围增大. 此外, CT扫描还可以发 现肺的过度膨胀, 定量 $\mathrm{CT}$ 扫描将肺组织密度值低于 $-900 \mathrm{HU}$ 定义为过度膨胀, 高达44\%的ARDS患者可能 存在肺过度膨胀, 主要位于腹侧和非重力依赖区. 肺过 度膨胀是机械通气患者呼吸机导致肺损伤的独立危险 因素, 可以导致纤维化和支气管扩张发生.

$\mathrm{CT}$ 具有明显优于 CXR 的特点, 不仅可以发现 ARDS时肺部的形态学变化, 也可以进行呼吸功能的 评估, 但CT目前无法实现床旁监测. 实时评估肺部情 况需频繁转运患者, 转运、检查成本高且存在大剂量 放射线辐射风险，这限制了CT在肺部功能评估方面的 应用.

\section{3 经胸肺部超声}

经胸肺超声(lung ultrasound, LUS)是一种没有放 
射性、无创、易操作、可重复的床边技术．相比于听 诊和CXR, LUS可以更加精准地诊断肺实变、胸腔积 液、肺泡间质综合征和气胸. 正常情况下，充气的解 剖结构不传输超声波, 胸膜外看不到肺实质. 因此, 超 声下看到肺实质图像总是预示着病变. 基本的肺部超 声征象包括A线、 $\mathrm{B}$ 线和实变. ARDS典型超声表现为 不均匀分布的各种征象, 即重力依赖区实变为主，中 间区域的间质和肺泡水肿共存，表现为不同类型的B 线, 而非重力依赖区可表现为正常通气的 $\mathrm{A}$ 线. 临床试 验发现，机械通气患者中，通过9个点的LUS评估结果 与CT下的表现非常一致 ${ }^{[22]}$; 最近一项基于LUS的改良 ARDS柏林定义也被用于评估资源受限情况下ARDS 的发病率和预后 ${ }^{[23]}$.

与 CT类似, LUS可以进行肺功能的实时评估. Stefanidis等人 ${ }^{[24]}$ 观察到随着PEEP的增加，依赖区不通气 区域的减少和氧合的增加，并且显示出观察者之间的 良好一致性. 肺部的再充气评分对包含依赖区和非依 赖区各个重点部位的12个感兴趣区域进行LUS检查, 检测RM或调整PEEP等干预后超声模式的变化，并将 其变化进行定量，最后计算 12 个区域的平均LUS再充 气分数. 该评分不仅考虑了实变区域，而且还考虑了 中度和重度通气不良的肺区，对肺通气进行半定量评 估，与定量CT和经肺热稀释测量的血管外肺水(extravascular lung water, EVLW)结果具有良好的相关性, 通过LUS计算的肺通气评分预测肺复张的效能类似于 压力-容积曲线分析 ${ }^{[25]}$. 北京协和医院重症医学科王小 亭等人 ${ }^{[26]}$ 更深入地研究发现, ARDS患者利用俯卧位 肺部超声检查方案(prone position lung ultrasound examination, PLUE)在俯卧位 $0,3,6$ h进行背部 16 个区域 的检查, 得出再充气评分, 由此得出 $0 \sim 3 \mathrm{~h}$ 再充气评分 变分变化 (aeration score variation, ASV) $\geq 5.5$ 时, 预测患 者俯卧位潜能具有较高的敏感性和特异性 $(73.9 \%$, $86.4 \%$ ), 并且 $A S V \geq 7$ 显示出与预后的良好相关性(area under the receiver operating characteristic curve (AUROC) $0.702, P<0.01)$.

LUS 虽然能同时床旁实时呈现形态和功能的评 估, 但也有一定的局限性. 首先, LUS缺乏肺部的整体 评估, 并且超声成像很大程度上依赖伪影, 可能存在误 判, 尤其是在胸壁较厚, 成像质量较差的患者, 难以实 现定量分析，对皮下气肿和严重肺部创伤、烧伤患者 甚至可能会阻止超声束传播到肺表面; 其次，LUS对
于肺过度膨胀的评价价值不高; 最后，虽然LUS 是一 种高度可重复的技术, 但也不能作为一种持续的监测 工具.

\section{4 胸部电阻抗断层成像}

胸部电阻抗断层成像(electrical impedance tomography EIT)作为一种床旁无创、连续、动态、无辐射 的肺通气监测技术, 是一种功能成像技术, 主要通过局 部电极施加微弱电流, 感应通气过程中胸腔生物电阻 抗的变化，再利用相应的成像算法来动态实时评价肺 通气状态 ${ }^{[27]}$. EIT的时间分辨率很高 (监测频率可达 $25 \mathrm{~Hz}$, 相当于 $1 \mathrm{~s}$ 可以提供 25 帧肺通气的图像), 可有效 实现对区域性肺通气分布、肺容积、呼吸力学特征等 连续动态的监测, 有助于临床医生在床边更加精确地 观测到真实的肺通气和肺容积的变化情况, 有助于指 导ARDS肺复张、滴定PEEP，进而实施肺保护通气策 略，减少肺损伤. 典型的胸部EIT是通过在第三和第六 胁间胸壁放置电极带获得, 得到的图像代表了该部位 胸部横截面上发生的阻抗变化, 厚度通常可达 $5 \sim 10 \mathrm{~cm}$.

$\mathrm{EIT}$ 被广泛用于指导 ARDS 的机械通气设置. Grychtol等人 ${ }^{[28]}$ 提出了一种基于模糊逻辑的算法来分 析在平均气道压变化过程中获得的EIT图像, 以识别塌 陷区域的肺泡复张、功能正常区域的过度膨胀、之前 开放肺泡的塌陷和过度膨胀肺泡的恢复. 通过评价肺 内气体分布，可以指导潮气量、 PEEP的设置以及 RM 等操作. 在区域可复张性评估方面, EIT明显优于其他 影像学方法, 在增减PEEP试验中, EIT可以发现增加背 部通气的动作，而高压力支持水平、高潮气量与腹侧 通气增加和非依赖区肺过度牵张相关. EIT衍生的“潮 气内气体分布”和“过度膨胀”等指标以及局部通气延 迟指数 ${ }^{[29]}$ 、 Pendelluft现象, 被证明是监测肺损伤的非 常有前景的指标 ${ }^{[27]}$.

EIT应用于肺血流分布的监测也是目前研究热点 之一. “气来血来, 气走血走”是生命呼吸的基本原则, 肺脏是通气的器官, 同时又是接受 $100 \%$ 心输出量的通 血器官. 新冠肺炎(coronavirus disease 2019, COVID19)L型患者异于常规ARDS的临床表现，使大家意识 到肺血流异常在发病过程中的重要作用 ${ }^{[30]}$, 因此肺血 流的评估和干预在ARDS治疗中起至关重要的作用. 与灌注相关的胸部电阻抗变化比通气引起的变化小一 
个数量级, 因此提取有关肺灌注的信息要困难得多. EIT通过两种测量阻抗变化的方法对肺灌注进行研究, 一种方法通过肺的血流搏动法，另一种是通过注射一 种比肺组织具有更高导电性的对比剂, 比如高渗盐水 进行造影, 基于在呼吸屏气时注射造影剂产生的电阻时间曲线评估肺血流灌注分布. 盐水造影EIT肺血流成 像比血流搏动法准确性更高, 受到人们广泛关注 ${ }^{[31]}$. 北京协和医院重症医学科对该技术进行较深入的研 究, 积累较丰富的经验, 率先制定了相关技术规范 ${ }^{[31]}$, 应用EIT盐水造影技术, 在ARDS患者发现高PEEP引起 的区域性肺泡过度膨胀和复张与区域 $\mathrm{V} / \mathrm{Q}$ 的变化有关. 过渡膨胀/复张 $(\mathrm{O} / \mathrm{R})$ 指数 $<0.15$ 提示高PEEP可改善通 气的均一性, 提高通气血流匹配程度可能性大, 是潜在 指导PEEP设置的指标 ${ }^{[32]}$. 此外, 北京协和医院还用该 技术对肺栓塞(pulmonary embolism, PE)诊断进行了探 索, 在一项前瞻性概念性临床研究中, 重症医学科隆云 团队 ${ }^{[33]}$ 发现，基于EIT浓盐水造影获得的指标——死 腔通气分数(Dead Space\%)诊断PE的性能优于血清D二聚体(AUC: 0.986 vs. $0.502, P<0.05$ ). 以EIT Dead Space $\%>30.37 \%$ 诊断PE的灵敏度为 $90.9 \%$, 特异度为 98.6\%.

此外, EIT也被用于新冠肺炎的临床研究. 基于 COVID-19呼吸衰竭的病理生理特点, 可以将其分为 $\mathrm{H}$ 型和 $\mathrm{L}$ 型，两者对呼吸支持的反应不同，肺通气和肺灌 注具有重要意义. 有小样本研究评价了EIT在COVID19 相关ARDS患者中的应用，发现肺可复张性存在明 显异质性，存在高死腔的病理特征, EIT可为COVID19 患者选择个体化的最佳PEEP ${ }^{[34]}$. 目前也有研究应用 EIT盐水造影肺灌注成像评估COVID-19患者的肺灌 注, 结合 $\mathrm{V} / \mathrm{Q}$ 综合制定治疗方案 ${ }^{[35]}$.

EIT可以实时、连续地在床边评估肺通气的分布 以及肺血流的评估, 对重症患者机械通气参数的优化 具有重要意义, 但EIT是一种单纯的功能学评估手段, 其结果受绑带位置、胸遊、心脏、膈肌等结构和位置 的影响，增加监测层面或三维成像方法目前尚不成熟， 因此需结合其他形态学监测方法实现对肺脏的全面 评估.

\section{ARDS影像学监测: 从肺保护到循环保护}

近几十年的实践证实，机械通气在治疗ARDS的
同时，不仅可以导致呼吸机相关性肺损伤，也会对循 环产生重大影响 ${ }^{[7]}$. ARDS患者循环衰竭发生率高达 $70 \%$ ，大多数ARDS患者并非死于严重肺损伤和顽固 性低氧血症, 而是死于休克和突发心跳骤停, 因此在 当前的机械通气策略中仅强调肺保护避免肺损伤可能 还远远不足, 早在2016年, 北京协和医院重症医学科隆 云和刘大为 ${ }^{[36]}$ 就率先提出了ARDS的循环保护通气策 略, 即临床医疗不应仅关注呼吸力学对肺脏的影响, 还 应关注呼吸运动产生的血流动力学效应, 强调需要关 注机械通气对静脉回流、右心后负荷、血管张力、组 织灌注等方面的影响，在开放肺、保护肺的同时应避 免机械通气对循环的损伤, 将ARDS机械通气策略从 “肺保护”深入到“循环保护”, 实施以血流动力学评估 和血流动力学治疗为导向的循环保护性通气策略. ARDS的影像监测, 也从早期侧重肺通气本身的监测 深入到肺血流等循环相关的监测.

新冠肺炎L型患者肺顺应性好, 通气良好, 但仍存 在严重低氧血症，这种异于常规的临床表现使人们深 刻认识到肺血流分布异常(分流)导致的 $\mathrm{V} / \mathrm{Q}$ 失调在病 理机制中的作用, 临床上肺血流灌注评估主要依赖 CT 肺血管造影或核素显像等检查完成，但存在放射性 高、费时且需要转运到特定检查场所完成等要求, 在 重症患者中开展受到一定制约。EIT下血流成像技 术 ${ }^{[31]}$ 被应用于评估重症新冠患者肺血流灌注分布，有 效解决了重症新冠患者床旁肺血流评估的难题, 为新 冠重症患者提供肺血流和通气区域分布、区域 $\mathrm{V} / \mathrm{Q}$ 的 测量、临床指导PEEP的设置和俯卧位通气治疗 ${ }^{[35]}$.

ARDS本身的严重缺氧、肺泡毛细血管膜渗出引 起肺循环阻力升高, 肺高压继而引起右心后负荷增加, 机械通气的目的是复张塌陷的肺泡, 增加功能残气量, 纠正低氧血症, 降低肺循环阻力, 改善右心后负荷, 但 由于ARDS时肺内病变的不均一, 不恰当的PEEP和潮 气量设置并没有开放塌陷肺泡, 反而导致正常区域的 肺泡过度膨胀, 进一步增加右心后负荷, 降低心输出 量及恶化血流动力学. 肺泡的过度膨胀和不张在EIT 下可以直观显示出来 ${ }^{[27]}$, 超声心动图则可以直观显示 右心室，右心室急性扩张，右室与左室舒张末期内径 比值 $>0.6$, 并且收缩末期室间隔矛盾运动, 即可诊断急 性肺心病(acute cor pulmonale, ACP) ${ }^{[37]}$. ACP在中重度

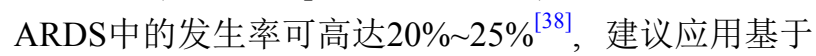
超声的心肺交互作用评估来指导ARDS患者ACP的预 
防及早期发现，采用低驱动压、限制性高碳酸血症和 维持肺开放的合适PEEP等循环保护通气策略和不增 加气道平台压的俯卧位来治疗 ACP, 并且强调连续的 右心功能评估来指导ARDS机械通气治疗策略及强 度 $^{[36,38,39]}$.

\section{6 其他}

一些功能显像技术也被用于ARDS的评估. 正电 子发射断层扫描(positron emission tomography, PET) 是一种功能成像技术，它是基于标记有放射性同位素 的分子随着正电子的发射而衰变, 来反映炎症环境中 炎症细胞的存在和活性. 它可以很早期发现正常充气 肺组织的异常炎症反应，对了解肺影像与肺损伤之间 的关系作出了重大贡献，是对现有形态学成像技术的 良好补充. 磁共振成像(magnetic resonance imaging, $\mathrm{MRI}$ )也是一种功能性技术, 为研究肺部疾病的病理生 理学提供了可能. 超极化惰性气体磁共振成像可以提
供高空间分辨率的通气灌注不均匀性、肺末梢毛细血 管氧扩散和肺微结构的信息. 最近的研究表明, 可以根 据MRI的表观扩散系数计算肺泡大小, 用来评估肺不 张和肺泡复张的效果 ${ }^{[4,40]}$.

\section{7 总结与展望}

肺部影像学可能是更好地理解ARDS这一综合征 最好的方法, 从形态学检查到功能评估, 影像学的发展 促进了人们对ARDS的认识和治疗措施的改进. 除了 需要转运的经典放射学技术CXR、CT外, 胸部超声和 EIT等新技术可以在床旁提供一种简洁、无创、实时 动态的方法来进行ARDS的监测, 以形态监测的目的 实现功能评估，导向目标治疗. 肺部形态结合功能评 估是目前ARDS评估必不可少的两个方面, 未来ARDS 的影像学一方面朝着更加便捷的形态评估发展, 另一 方面也朝着更加复杂的可以帮助人们观察肺部血流情 况、理解病理生理机制的整体功能评估发展.

\section{参考文献}

1 Liu D W. Acute respiratory distress sydrome: Past and Present (in Chinese). Med J Peking Union Med Coll Hosp, 2020, 11: 501-507 [刘大为. 急 性呼吸窘迫综合征: 前世今生. 协和医学杂志, 2020, 11: 501-507]

2 Fan E, Brodie D, Slutsky A S. Acute respiratory distress syndrome: Advances in diagnosis and treatment. JAMA, 2018, 319: 698-710

3 Sweeney R M, McAuley D F. Acute respiratory distress syndrome. Lancet, 2016, 388: 2416-2430

4 Pesenti A, Musch G, Lichtenstein D, et al. Imaging in acute respiratory distress syndrome. Intensive Care Med, 2016, 42: 686-698

5 Ashbaugh D G, Boyd Bigelow D, Petty T L, et al. Acute respiratory distress in adults. Lancet, 1967, 290: 319-323

6 Ferguson N D, Fan E, Camporota L, et al. The Berlin definition of ARDS: an expanded rationale, justification, and supplementary material. Intensive Care Med, 2012, 38: 1573-1582

7 Thompson B T, Chambers R C, Liu K D. acute respiratory distress syndrome. N Engl J Med, 2017, 377: 562-572

8 Bellani G, Laffey J G, Pham T, et al. Epidemiology, patterns of care, and mortality for patients with acute respiratory distress syndrome in intensive care units in 50 countries. JAMA, 2016, 315: 788-800

9 Herasevich V, Yilmaz M, Khan H, et al. Validation of an electronic surveillance system for acute lung injury. Intensive Care Med, 2009, 35: 1018-1023

10 Lichtenstein D, Goldstein I, Mourgeon E, et al. Comparative diagnostic performances of auscultation, chest radiography, and lung ultrasonography in acute respiratory distress syndrome. Anesthesiology, 2004, 100: 9-15

11 Xirouchaki N, Magkanas E, Vaporidi K, et al. Lung ultrasound in critically ill patients: comparison with bedside chest radiography. Intensive Care Med, 2011, 37: 1488-1493

12 Wallet F, Delannoy B, Haquin A, et al. Evaluation of recruited lung volume at inspiratory plateau pressure with PEEP using bedside digital chest X-ray in patients with acute lung injury/ARDS. Respir Care, 2013, 58: 416-423

13 Gattinoni L, Presenti A, Torresin A, et al. Adult respiratory distress syndrome profiles by computed tomography. J Thorac Imag, 1986, 1: 25-30

14 Wang L H, Pan C, Huang Y Z. Videography effect of RM on the heterogeneity of ventilation in ARDS (in Chinses). Chin J Crit Care Intensive Care Med, 2018, 4: 285-288 [王丽晖, 潘纯, 黄英姿. 急性呼吸窘迫综合征肺内气体分布不均一性的影像学监测技术进展. 中华重症医学电 
子杂志, 2018, 4: 285-288]

15 Gattinoni L, Caironi P, Pelosi P, et al. What has computed tomography taught us about the acute respiratory distress syndrome? Am J Respir Crit Care Med, 2001, 164: 1701-1711

16 Wang S, Ruan Z, Zhang J, et al. The value of pulmonary contusion volume measurement with three-dimensional computed tomography in predicting acute respiratory distress syndrome development. Ann Thorac Surg, 2011, 92: 1977-1983

17 Strumwasser A, Chu E, Yeung L, et al. A novel CT volume index score correlates with outcomes in polytrauma patients with pulmonary contusion. J Surg Res, 2011, 170: 280-285

18 Yoshida T, Rinka H, Kaji A, et al. The impact of spontaneous ventilation on distribution of lung aeration in patients with acute respiratory distress syndrome: airway pressure release ventilation versus pressure support ventilation. Anesth Analg, 2009, 109: 1892-1900

19 Malbouisson L M, Muller J C, Constantin J M, et al. Computed tomography assessment of positive end-expiratory pressure-induced alveolar recruitment in patients with acute respiratory distress syndrome. Am J Respir Crit Care Med, 2001, 163: 1444-1450

20 Gattinoni L, Caironi P, Cressoni M, et al. Lung recruitment in patients with the acute respiratory distress syndrome. N Engl J Med, 2006, 354: $1775-1786$

21 Cressoni M, Cadringher P, Chiurazzi C, et al. Lung inhomogeneity in patients with acute respiratory distress syndrome. Am J Respir Crit Care Med, 2014, 189: 149-158

22 Tierney D M, Huelster J S, Overgaard J D, et al. Comparative performance of pulmonary ultrasound, chest radiograph, and CT among patients with acute respiratory failure. Crit Care Med, 2020, 48: 151-157

23 Riviello E D, Kiviri W, Twagirumugabe T, et al. Hospital incidence and outcomes of the acute respiratory distress syndrome using the kigali modification of the Berlin definition. Am J Respir Crit Care Med, 2016, 193: 52-59

24 Stefanidis K, Dimopoulos S, Tripodaki E S, et al. Lung sonography and recruitment in patients with early acute respiratory distress syndrome: A pilot study. Crit Care, 2011, 15: R185

25 Bouhemad B, Brisson H, Le-Guen M, et al. Bedside ultrasound assessment of positive end-expiratory pressure-induced lung recruitment. Am J Respir Crit Care Med, 2011, 183: 341-347

26 Wang X T, Ding X, Zhang H M, et al. Lung ultrasound can be used to predict the potential of prone positioning and assess prognosis in patients with acute respiratory distress syndrome. Crit Care, 2016, 20: 385

27 Bachmann M C, Morais C, Bugedo G, et al. Electrical impedance tomography in acute respiratory distress syndrome. Crit Care, 2018, 22: 263 Grychtol B, Wolf G K, Adler A, et al. Towards lung EIT image segmentation: automatic classification of lung tissue state from analysis of EIT monitored recruitment manoeuvres. Physiol Meas, 2010, 31: S31-S43

29 Muders T, Luepschen H, Zinserling J, et al. Tidal recruitment assessed by electrical impedance tomography and computed tomography in a porcine model of lung injury. Critical Care Med, 2012, 40: 903-911

30 Gattinoni L, Chiumello D, Rossi S. COVID-19 pneumonia: ARDS or not? Crit Care, 2020, 24: 154

31 He H W, Long Y, Chi Y, et al. Technology specification of bedside hypertonic saline-contrast electrical impedance tomography of lung perfusion and clinical application (in Chinese). Natl Med J China, 2021, 101: 1097-1101 [何怀武, 隆云, 池熠, 等. 床旁高渗盐水造影肺灌注电阻抗断层 成像的技术规范与临床应用. 中华医学杂志, 2021, 101: 1097-1101]

$32 \mathrm{He} \mathrm{H}$, Chi Y, Long Y, et al. Influence of overdistension/recruitment induced by high positive end-expiratory pressure on ventilation-perfusion matching assessed by electrical impedance tomography with saline bolus. Crit Care, 2020, 24: 586

33 He H, Long Y, Frerichs I, et al. Detection of acute pulmonary embolism by electrical impedance tomography and saline bolus injection. Am J Respir Crit Care Med, 2020, 202: 881-882

34 Mauri T, Spinelli E, Scotti E, et al. Potential for lung recruitment and ventilation-perfusion mismatch in patients with the acute respiratory distress syndrome from coronavirus disease 2019. Crit Care Med, 2020, 48: 1129-1134

35 Perier F, Tuffet S, Maraffi T, et al. Effect of positive end-expiratory pressure and proning on ventilation and perfusion in COVID-19 acute respiratory distress syndrome. Am J Respir Crit Care Med, 2020, 202: 1713-1717

36 Long Y, Liu D W. The conception of circulation protective ventilation strategy (in Chinese). Chin J Intern Med, 2016, 55: 172-174 [隆云, 刘大 为. 循环保护性通气策略的提出与内涵. 中华内科杂志, 2016, 55: 172-174]

37 Guérin C, Matthay M A. Acute cor pulmonale and the acute respiratory distress syndrome. Intensive Care Med, 2016, 42: 934-936

38 Vieillard-Baron A, Matthay M, Teboul J L, et al. Experts' opinion on management of hemodynamics in ARDS patients: focus on the effects of 
mechanical ventilation. Intensive Care Med, 2016, 42: 739-749

Wang X T, Liu D W, Zhang H M, et al. Expert consensus on the management of the right heart function in critically ill patients (in Chinese). Chin J Intern Med, 2017, 56: 962-973 [王小亭, 刘大为, 张宏民, 等. 重症右心功能管理专家共识. 中华内科杂志, 2017, 56: 962-973]

40 Chiumello D, Froio S, Bouhemad B, et al. Clinical review: Lung imaging in acute respiratory distress syndrome patients - an update. Crit Care, 2013, 17: 243

\title{
Advances on acute respiratory distress syndrome based on imaging: from morphology to function
}

\author{
CHENG Wei ${ }^{1}$, HE HuaiWu ${ }^{1}$, SU LongXiang ${ }^{1}$, CUI Na ${ }^{1}$, WANG XiaoTing ${ }^{1}$, ZHOU Xiang $^{1}$, \\ WENG $\mathrm{Li}^{2}$, LONG Yun ${ }^{1}$, DU Bin $^{2} \&$ LIU DaWei ${ }^{1}$ \\ 1 Department of Critical Care Medicine, State Key Laboratory of Complex Severe and Rare Diseases, Peking Union Medical College Hospital, \\ Chinese Academy of Medical Sciences \& Peking Union Medical College, Beijing 100730, China; \\ 2 Department of Medical Critical Care Medicine, State Key Laboratory of Complex Severe and Rare Diseases, Peking Union Medical College \\ Hospital, Chinese Academy of Medical Sciences \& Peking Union Medical College, Beijing 100730, China
}

Controversy and challenges have accompanied acute respiratory distress syndrome (ARDS) since it was proposed 50 years ago. The progress of lung imaging has witnessed the development of ARDS. From the earliest chest X-ray to chest computed tomography (CT), to non-radioactive, bedside chest ultrasound, electrical impedance imaging and other methods, lung imaging plays an important role in the diagnosis and treatment of ARDS and the research of pathophysiological mechanism. The development of ARDS imaging progresses from whole to local, from pulmonary ventilation to blood perfusion, from simple respiratory assessment to combination with circulatory assessment, and from morphological analysis to functional assessment. Till now, ARDS imaging is not only used in diagnosis, but also in bedside continuous evaluation to guide treatment. This article systematically reviews the progress of clinically available imaging evaluation of ARDS, hoping to better understand the morphological and functional changes of ARDS, to guide clinical treatment, and to improve the prognosis of these patients.

acute respiratory distress syndrome, chest X-ray, chest computed tomography, lung ultrasound, electrical impedance tomography

doi: $10.1360 /$ SSV-2021-0201 